\title{
On Cobb angles, flexibility, and lumbar strength measurements
}

\author{
Jeb McAviney ${ }^{1}$, Benjamin T. Brown ${ }^{2, *}$ \\ ${ }^{1}$ Sydney Scoliosis Clinic, Kogarah, Australia \\ ${ }^{2}$ Macquarie University, North Ryde, Australia
}

To the editor:

The authors would like to thank Ko and Kang for their contribution to the literature in their article titled "Effects of 12-week core stabilization exercise on the Cobb angle and lumbar muscle strength of adolescents with idiopathic scoliosis" (Ko and Kang, 2017). However, we believe that the conclusions drawn by these authors are not adequately supported by their study findings.

Research into the effects of exercise as an intervention for adolescent idiopathic scoliosis (AIS) is, without question, an important pursuit given the impact that this condition has on patients, and the direct and indirect costs involved with the management of AIS. Research into this condition serves to inform both practitioners and patients of treatment options which can range from conservative strategies, e.g., exercise, all the way through to highly invasive surgical procedures.

In Ko and Kang's study, radiographic Cobb angles, flexibility and lumbar strength measurements were taken in a group (control and intervention) of primary school students before and after a 12week core stabilization exercise program. The results from a control group were compared to those in the exercise group. The authors report that there were significant changes in the Lumbar Cobb angles of the exercise group. The figures that have been presented in Table 2 of the manuscript indicate that the preintervention mean and standard deviation lumbar Cobb angle in the exer- cise group was $15.95^{\circ} \pm 1.84^{\circ}$, and then $15.21^{\circ} \pm 1.91^{\circ}$ after 12 weeks. Using a repeated-measure two-way analysis of variance the authors reported statistically significant differences (alpha $=0.05$ ) in these pre- and post-Cobb angles. The authors then went on to conclude that core stabilization exercise is effective for reducing the Cobb angle in patients with AIS.

Given that the measurement error for radiographic Cobb angle measurement (Langensiepen et al., 2013; Morrissy et al., 1990) and diurnal variation (Beauchamp et al., 1993) may be as high as $5^{\circ}$, and the minimal clinically important difference is considered to be $\geq 5^{\circ}$ (Carman et al., 1990), it is difficult to understand how Ko and Kang can make such strong statements regarding efficacy. Furthermore, the study is not a randomised controlled trial which further limits the strength of the authors' conclusions.

We would recommend that Ko and Kang revise their conclusions to more closely align with the robustness of their research findings and the inherent limitations associated with their chosen research design so as not to confound the evidence in this important area.

\section{CONFLICT OF INTEREST}

No potential conflict of interest relevant to this article was reported.
*Corresponding author: Benjamin T. Brown (i) https://orcid.org/0000-0002-3064-8815 Macquarie University, Balaclava Rd, North Ryde, NSW 2109, Australia

Tel: +61-2-8006-0656, Fax: +61-2-8212-9059, E-mail: benjamin.brown@mq.edu.au Received: June 27, 2017 / Accepted: August 13, 2017
This is an Open Access article distributed under the terms of the Creative Commons Attribution Non-Commercial License (http://creativecommons.org/licenses/by-nc/4.0/) which permits unrestricted non-commercial use, distribution, and reproduction in any medium, provided the original work is properly cited. 


\section{REFERENCES}

Beauchamp M, Labelle H, Grimard G, Stanciu C, Poitras B, Dansereau J. Diurnal variation of Cobb angle measurement in adolescent idiopathic scoliosis. Spine (Phila Pa 1976) 1993;18:1581-1583.

Carman DL, Browne RH, Birch JG. Measurement of scoliosis and kyphosis radiographs. Intraobserver and interobserver variation. J Bone Joint Surg Am 1990;72:328-333.

Ko KJ, Kang SJ. Effects of 12-week core stabilization exercise on the Cobb angle and lumbar muscle strength of adolescents with idiopathic scoliosis. J Exerc Rehabil 2017;13:244-249.

Langensiepen S, Semler O, Sobottke R, Fricke O, Franklin J, Schönau E, Eysel $\mathrm{P}$. Measuring procedures to determine the Cobb angle in idiopathic scoliosis: a systematic review. Eur Spine J 2013;22:2360-2371.

Morrissy RT, Goldsmith GS, Hall EC, Kehl D, Cowie GH. Measurement of the Cobb angle on radiographs of patients who have scoliosis. Evaluation of intrinsic error. J Bone Joint Surg Am 1990;72:320-327. 